\title{
Female urinary incontinence: is laser treatment effective? a systematic review
}

\begin{abstract}
Background: Towards the recent promising results of microablative fractional $\mathrm{CO}_{2}$-laser and non-ablative photothermal Erbium: YAG-laser (Er:YAG-laser) as therapy performed in Gynaecology; this systematic review aims to summarize the effectiveness and safety of both laser technologies on female Urinary Incontinence (UI).

Methods: Using "laser" and "urinary incontinence" as query, studies were searched from MEDLINE and SCOPUS up to February 2018. Information from seventeen studies, counting more than 892 female patients with UI, was systematically registered. 652 and more than 240 patients were treated with Er:YAG-laser and $\mathrm{CO}_{2}$-laser, respectively.

Results: Results addressing patient reported outcomes through different validated questionnaires showed statistically significant improvement in UI after Laser therapy. Pad weight tests, perineometry, Q-tip test, urodynamics and epithelial biopsies as assessment to objective quantification of UI and pelvic structures remodeling also showed improvement but these results were not so consistent. In general, laser therapy was well tolerated and no major side effects were reported.
\end{abstract}

Conclusion: Laser therapy seems to be a promising alternative approach to UI in women. However, there are no randomized controlled trials in this field and so the level of evidence is still low. More research is required to further apply these technologies in women safely and effectively.

Keywords: laser, urinary incontinence, gynaecology, review
Volume 9 Issue 4 - 2018

\author{
João Henriques,' Pedro Brandão, ${ }^{1,2}$ Amélia \\ Almeida, ${ }^{3,4}$ Paula Ramôa ${ }^{4}$ \\ 'Faculty of medicine, University of Porto, Portugal \\ ${ }^{2}$ Department of Obstetrics and Gynaecology, Centro Hospitalar \\ Tâmega e Sousa, Portugal \\ ${ }^{3}$ Department of Obstetrics and Gynaecology, Centro Hospitalar \\ Medio Ave, Portugal \\ ${ }^{4}$ Department of Obstetrics and Gynaecology, Hospital Lusiadas \\ Porto, Portugal
}

\begin{abstract}
Correspondence: Pedro Brandão, Department of Obstetrics and Gynaecology, Centro Hospitalar Tâmega e Sousa, Avenida do Hospital Padre Américo 2 10, 4564-007 Guilhufe, Portugal, Tel 255-7|4-000, Email pedrobrandaoleite@gmail.com
\end{abstract}

Received: June 08, 2018 | Published: July 05, 2018

\section{Introduction}

Urinary incontinence (UI) was defined by the International Urogynecological Association (IUGA) and the International Continence Society (ICS) as involuntary urine loss. Regarding the underlying pathophysiological mechanism, UI can be divided into stress UI (SUI) - UI on effort, physical exertion, sneezing or coughing; urgency UI (UUI) - associated with urgency; and mixed UI (MUI) - when both are present. ${ }^{1,2}$ It affects young and old women and due to its high prevalence has a significant impact on the psychological and socioeconomic aspects of life. ${ }^{3,4}$ There is a wide range of therapeutic options for UI, both medical and surgical, but new and minimally invasive methods have recently been introduced to optimize efficacy and overcome the complications and morbidity of standard therapies. ${ }^{1,5}$

Laser therapy has a therapeutic role in various medical conditions and most recently has gained interest as a promising treatment for UI, particularly SUI, the most prevalent type of UI. ${ }^{1,6}$ In this field, two distinct lasers have been used: microablative fractional $\mathrm{CO}_{2}-$ laser $\left(\mathrm{CO}_{2}\right.$-laser $)$ and non-ablative photothermal Erbium: YAG-laser (Er:YAG-laser). ${ }^{7}$

The $\mathrm{CO}_{2}$-laser was the first laser being developed and used in its microablative fractional mode as vaginal treatment for the genitourinary syndrome of menopause (GSM) and female UI. The mechanism behind this therapy it's related to the light emission at $10.600 \mathrm{~nm}$, which is absorbed by the water contained in vaginal mucosal tissues. ${ }^{6}$ This produces a thermal effect that stimulates tissues to synthetize collagen besides other molecular changes. The posttreatment effect of this neocollagenesis is newly formed connective tissue, recovery of sub mucosal capillaries as well as an increased thickness of the epithelium. This explains the restoration of most vaginal functions and also an increase in muscle tone that seems to be related to a clinical improvement in UI. $6,8,9$

The thermal effect achieved with the $\mathrm{CO}_{2}$-laser occurs at depths of about $50-125 \mu \mathrm{m}$ in the vaginal tissue. On the other hand we have a 5-20 $\mu \mathrm{m}$ deep incremental thermal effect produced by the Er:YAGlaser, not causing superficial vaporization unlike $\mathrm{CO}_{2}$-laser, which makes it a true nonablative laser. ${ }^{9}$ The use of Er:YAG-laser in a nonablative mode enables thermal-only operation - at $60-65^{\circ} \mathrm{C}$ - that causes shrinkage of collagen fibers without irreversible denaturation of their structure. ${ }^{9-11}$ There is immediate shrinkage of the upper layers of the vaginal wall as well as mechanical pull of the lower lying structures achieved by the laser thermal effect. But this effect is not just momentary during laser exposure, the collagen remodelling and neocollagenesis processes continue during approximately 6 months after laser session. ${ }^{11}$

Towards the promising results of non-ablative Er:YAG-laser on UI, many additional studies have been initiated to further assess this technology and treatment approach. ${ }^{6}$ Even though there is currently a reasonable number of studies about vaginal $\mathrm{CO}_{2}$-laser and Er:YAGlaser treatments, there are no reviews focusing on both kinds of laser as an approach to any type of female UI (SUI, UUI and MUI). ${ }^{5,7,12}$ The aim of the present study is to identify the effectiveness and safety of both $\mathrm{CO}_{2}$-laser and Er:YAG-laser as therapy for female urinary incontinence by reviewing literature.

\section{Material and methods}

The present study was planned using the Preferred Reporting Items for Systematic Reviews and Meta-Analyses (PRISMA) guidelines. ${ }^{13}$ Studies that evaluate the impact of $\mathrm{CO}_{2}$-laser or Er:YAG-laser on women with UI was chosen. 
MEDLINE and SCOPUS electronic databases were accessed and articles published until February 2018 was retreived. The expressions "laser" and "urinary incontinence" were used as query in PubMed and SCOPUS. Case reports, animal studies and non-English studies were excluded.

The study selection process took place in two phases. In the initial search, titles and/or abstracts of all electronic articles were screened to assess eligibility by 2 of the authors independently. All original research studies concerning laser treatment on female UI were included. Male related studies, including the terms "prostate" and "prostatic", as well as studies involving surgical procedures, including the terms "laparotomy" and "laparoscopy" was excluded. Then, all articles that met or were presumed to meet criteria were retrieved as full texts to be examined. All included articles were examined and information was retrieved by the same 2 authors. Discordances between authors were solved by agreement. The search and selection process are shown in the flow diagram (Figure 1).

\section{IDENTIFICATION}

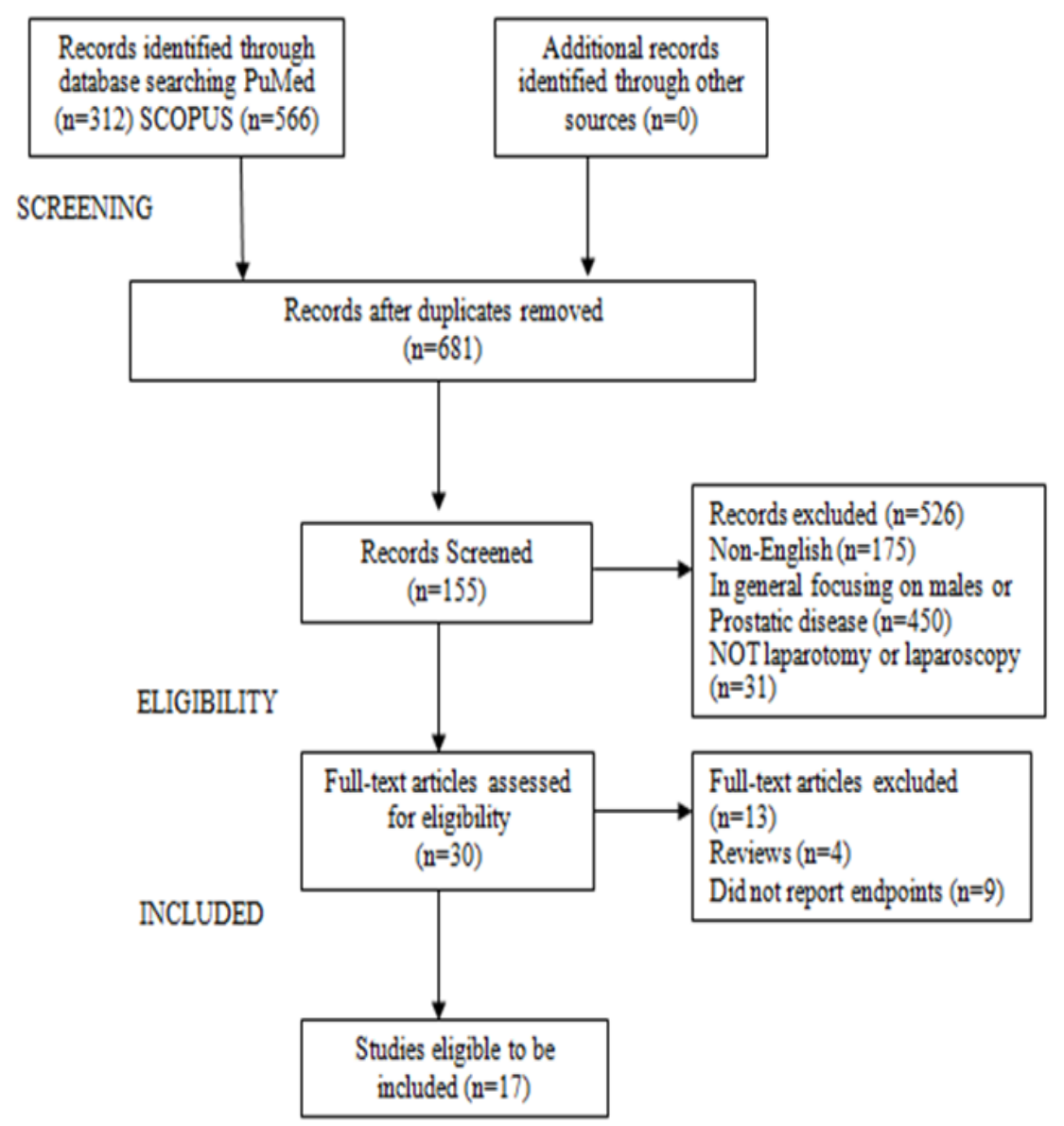

Figure I: Search method.

The primary outcome was the impact of laser therapy evaluated through patient reported outcomes (mostly validated questionnaires), pad weight test, Q-tip test, residual volume/ urodynamics, perineometry or histological features. Reported adverse effects and safety were secondary outcomes. Prospective studies involving laser treatment of urinary incontinence in women were selected. References of full text articles were also screened and used as additional information.

Information was systematically registered on: the number and main features (age, Body Mass Index (BMI) and parity) of the participants in each study; the inclusion and exclusion criteria; number of patients with each type of UI; details of the intervention (type of laser, additional therapies, number of sessions, follow-up timings); loss to follow-up, and primary and secondary outcomes.

\section{Results}

Seventeen studies counting more than 892 female patients with UI were included in the review. 4,8,10,14-27 The search of MEDLINE and SCOPUS provided a total of 878 records. After removing duplicates, 526 studies were excluded because they did not meet the criteria. 30 articles were examined in more detail. Of these, 17 were included to be analysed in this systematic review. The main characteristics of the included articles and outcomes are summarized (Table 1) (Table 2). 
All 17 studies selected are prospective non-comparative studies, and they all have in common as inclusion criteria women with UI alone or UI as part of GSM. On the opposite, the presence of severe prolapse, genital injury and/or active infection, as well as undiagnosed vaginal bleeding, abnormal cervical cytology and pregnancy were recurrently considered as exclusion criteria. The age, BMI and parity of the individuals were also taken into consideration in the majority of the studies. The minimal mean age registered was 43.3 and maximum 62.9 years; with medians between 46.5 and 56 years old. The average weight was normal or overweight, with maximum BMI mean of 27.5 $\mathrm{Kg} / \mathrm{m}^{2}$. When registered, the median parity of women was between 1 and 2 , with a range of $0-5$.

Twelve of the studies used the Er:YAG-laser and five the $\mathrm{CO}_{2}$ laser; corresponding to 652 and more than 240 patients treated with Er:YAG-laser and $\mathrm{CO}_{2}$-laser, respectively. The type of UI patients presented in each study was also taken in consideration as well as the number of laser sessions and the follow-up patients received: all women undertook at least 1 laser session and were evaluated before - baseline (B) - and at least one time after the treatment (F). Only 4 studies reported additional therapies, which included: supplements of Vitamins A and $\mathrm{C}$; prophylactic antibiotics; topical estrogens orsilicon-based balm; and local anaesthesia prior the procedure. ${ }^{4,14,19,23}$

The primary outcomes relevant for this review were the effects of laser therapy on UI. Patient reported outcomes were mainly evaluated through validated questionnaires. Objective measures of urine leakage and status of pelvic structures were based on weight pad tests, Q-tip test, urodynamics / residual volume, perineometry and biopsies.

\section{Patient reported outcomes}

Twelve studies used the International Consultation on Incontinence Questionnaire - Urinary Incontinence Short Form (ICIQ-UI SF), which is composed by three questions ("How often do you leak urine?"; "How much urine do you usually leak?"; "Overall, how much does leaking urine interfere with your everyday life?") and results in a sum-score between 0 and 21; with a cut-off of $\geq 1$ meaning UI and 0 meaning no UI. The sum-score may be divided into four severity categories: mild (1-5), moderate (6-12), severe (13-18), and very severe (19-21) UI. ${ }^{4,8,10,17-24,27}$

Three studies used the and Overactive Bladder Questionnaire Short Form (OAB-Q SF), which consists of 19 items comprised by two scales: a 6-item Symptom Bother scale and a 13-item healthrelated quality of life (HRQOL) scale..$^{14,16,21,28}$ The Urogenital Distress Inventory (UDI-6) was used in 3 studies and the Incontinence Impact Questionnaire (IIQ-7) in 2 studies, with 6 and 7 questions regarding urinary incontinence, respectively. ${ }^{14,17,21}$

Behnia et al. ${ }^{23}$ based their results merely in patients' subjective evaluation. Most studies present their results by mean with the standard deviation (mean $\pm \mathrm{SD}$ ) or median with the interquartile ranges (Median (IQR)) of scores.

Concerning Erbium-YAG laser, the first study published in 2015 by Ogrinc et al. ${ }^{10}$ found a significant improvement after treatment in $77 \%(n=88)$ of patients diagnosed with SUI six months after treatment. Patients with more severe UI and SUI alone had better improvement rates. On the other hand, age didn't affect the results and the results 12 months after treatment were not so consistent. This study had the largest patient's pool (175), and included patients with pure SUI and MUI. Gambacciani et al. ${ }^{20}$ reported a significant decrease in ICIQ-UI
SF score from basal values of $12.0 \pm 1.8$ to $5.6 \pm 2.6$ at the third laser session, and remained significantly lower than basal values at 4 weeks (5.5 \pm 2.6$), 12$ weeks $(5.5 \pm 2.9)$ as well as 24 weeks (5.0 \pm 2.6$)$ after laser sessions. Pardo et al. ${ }^{19}$ reported statistically significant lower median ICIQ-UI SF scores 3 to 6 months after (11 to 3). Most of the individuals reported improvement $(78.6 \%, \mathrm{n}=33)$ or a complete healing of SUI $(38.1 \%, \mathrm{n}=16)$, but a minority had no change $(16.7 \%$, $\mathrm{n}=7$ ) or got worse $(4.8 \%, \mathrm{n}=2)$. Gaspar et al. ${ }^{4}$ reported the cure of $64 \%(n=14)$ and $46 \%(n=10)$ patients, respectively at 3 and 6 months after treatment, $23 \%(\mathrm{n}=5)$ had no change and no patient got worse. In 2016, Fistonić et al. ${ }^{18}$ reported a significantly decreased ICIQ-UI SF score in all follow-up timings compared to baseline. The score was decreased on average by 6.3 points after 1 month, by 5.3 points after 2 months, and by 5.1 points 6 months after treatment. The same author had already found in 2015 statistically significant differences between baseline and 2-6 months after the intervention, with a total of $34 / 47(72.3 \%)$ of participants experimenting improvement and a decrease from 12 to 4 in median ICIQ-UI SF score..$^{15}$ In 2018, the same group published a study which, besides the improvement in median ICIQ-UI SF score from 12 to 6 in the same period of time, points age, BMI, basal Q-tip and ICIQ-UI SF scores as predictors of greater improvement. ${ }^{22}$ Tien et al. ${ }^{21}$ showed significant decrease, at 6 months follow-up, in OAB symptom score (4.1 \pm 2.8 to $2.7 \pm 2.6)$, UDI$6(4.1 \pm 2.9$ to $3.1 \pm 3.1)$, IIQ-7 score $(3.2 \pm 4.2$ to $2.7 \pm 4.9)$, PPBC score ( $2.7 \pm 1.0$ to $2.0 \pm 1.1)$ and USS score $(1.3 \pm 0.8$ to $0.8 \pm 0.7) .{ }^{14}$ Lin et al studied women with SUI alone or MUI and reported a mean decrease of 1, 1.2 and 1.8 in ICIQ-UI-SF, OAB symptom and UDI-6 scores, respectively, 3 months after treatment; but 12 months after there were no more statistically significant differences to baseline.

Regarding $\mathrm{CO}_{2}$-laser, González et al. ${ }^{8}$ study in 2017 had the longest follow-up period - 36 months - and reported statistically significant improvement in ICIQ-UI SF mean scores from $14.34 \pm 2.65$ at baseline to $7.09 \pm 1.1$ (12 months later) and to $6.76 \pm 0.82$ (36 months later). Pagano et al. ${ }^{27}$ had a shorter follow-up period -3 months - but showed also a significant reduction in ICIQ-UI SF mean score from $9.7 \pm 4.0$ to 7.1 \pm 4.2 . Both studies had exclusively women with SUI. On the other hand, concerning patients with UUI or urgency symptoms, Pitsouni et al. ${ }^{17}$ observed a significant reduction from $8.1 \pm 5.6$ to $3.4 \pm 4.3$ in mean ICIQ-UI SF score, from $3.6 \pm 2.4$ to $1.6 \pm 1.5$ in ICIQ-FLUTS score (Female Low Urinary Tract Symptoms), and from 30.2 \pm 21.4 to $11.90 \pm 16.6$ in UDI- 6 score, after a follow-up of 3 months. In OAB-symptoms score, Perino et al. ${ }^{16}$ also found a significant median decrease from 18.5(4.25) to $8(2.25) 30$ days after treatment. BehniaWillison also studied UUI, with a larger follow-up time (24 months) and found significant improvement in patient symptoms, but these results were based only on subjective evaluation of the patients. ${ }^{23}$

\section{Pad-weight tests}

Quantification of urine loss was performed by pad weight tests in 4 studies, in which participants performed provocation maneuvers and exercises during a 20-minutes or 1-hour period while wearing a preweighed perineal pad which was weighed again at the end. Based on the pad weight tests, UI may be classified as mild $(2-10 \mathrm{~g})$, moderate $(11-50 \mathrm{~g})$, or severe $(>50),{ }^{4,8,14,21}$ Tien et al. ${ }^{14}$ stated the cure of UI in $39.3 \%$ of participants, with a significant mean reduction of pad weight from $14.0 \pm 18.2 \mathrm{~g}$ to $6.1 \pm 13.1 \mathrm{~g}$ at 3 months. Gaspar et al. ${ }^{4}$ had similar findings with significant decrease in mean weight of $9 \mathrm{~g}$ after 6 months. Lin et al. ${ }^{21}$ showed a significant decrease from $13.2 \pm 17.7 \mathrm{~g}$ to $6.1 \pm 11.6 \mathrm{~g}$ one year later. González et al. ${ }^{8}$ reported significant changes 
in mean pad weight from $9.89 \pm 0.5 \mathrm{~g}$ at baseline to $3.52 \pm 1.89 \mathrm{~g}$ at 12 months, and there were no significant changes until the 36 months follow-up.

\section{Perineometry and urodynamics}

Fistonić studied perineometry after Er-YAG laser treatments and found no significant changes in 2016, but in 2018, with a larger set of patients, found significant improvement in perineal pressure (median change of 0.8 to $1.1 \mathrm{mmHg}$ ) and duration of effective contractions (median change of 3.6 seconds). These authors used also the Q-tip test to evaluate the degree of the urethra during valsalva maneuver with significant median reduction from $72.5(48.8-80)$ to $50(30-70)$ degrees. ${ }^{18,22}$

Concerning the residual urine volume, in 2018, the same group observed an improvement from median volume of $2.9(0.8-9.4)$ at baseline to $0.5(0-1.7), 6$ months after treatment.

The only study comparing urodynamics during laser treatments did not find significant changes. ${ }^{14}$

\section{Histological evaluation}

Four studies demonstrated changes in vaginal epithelium after laser treatment. ${ }^{825-27}$ There was significant increase in the thickness and staining of type III collagen of the epithelium, with higher population of intermediate and shedding superficial cells. ${ }^{8,27}$ Lappi et al quantified these changes, revealing significant increase in epithelium thickness $(114.19 \pm 17.31 \mu \mathrm{m}$ to $187.83 \pm 15.35 \mu \mathrm{m})$; volume density $(1.8 \pm 0.2 \%$ to $2.9 \pm 0.3 \%)$ and number of capillary profiles per test area $(8.5 \pm 0.63$ to $12.1 \pm 1.07)$ as well as increase proliferative activity based on Ki67 expression (19.05 $\pm 2.86 \%$ to $31.79 \pm 2.25 \%){ }^{25,26}$

\section{Adverse effects and safety}

The reported adverse effects and/or safety of laser sessions stand as the secondary outcome in this review. In all studies, the laser therapy was well tolerated or no major side effects were reported. Participants from five studies reported mild pain/discomfort, burning/warmth sensation, teasing/pricking, or irritation during laser sessions. . $^{10,15,18,19,21}$ After laser therapy, few women noticed increased vaginal discharge, slight vulvar edema, burning sensation, irritation, pelvic pain, dysuria, urinary tract infection, Herpes simplex breakout or vaginal spotting. These symptoms vanished spontaneously within $2 \mathrm{~h}$ to a maximum of 8 days. 19 patients had transient UUI after treatment. ${ }^{4,10,15,17,18,20,21}$ Only 1 patient was reported to quit treatments due to intolerance. ${ }^{20}$

\section{Discussion}

The use of laser technologies as treatment for women with UI seems a promising minimally invasive alternative to the current standard therapies. Specifically concerning SUI, as the most relevant type of UI, laser may provide improvement in patients' quality of life, being less invasive and with little complications compared to midurethral slings. However, the conclusions are poor due to the low level of evidence of the studies, the lack of randomized clinical trials (RCT) and the lack of studies comparing any laser approach to conventional UI treatments, such as pelvic floor exercises or midurethral slings. Additionally, there is still lack of evidence showing long-term safety and effectiveness which turns difficult to recommend and apply the use of laser therapy in clinical practice.

In the present systematic review, results have shown consistent short-term UI improvement, specifically when reported subjective measurements of HRQL based on patient reported outcomes. Validated questionnaires used to compare patient status before and after the procedure showed significant improvement in involuntary urine loss not only in terms of frequency and quantity, but also how much this problem affects patients' quality of life. The evidence is stronger in SUI than overactive bladder or UUI as most of the studies relied on the first. The majority of the patients improved with this technique, and only few studies reported quite few patients with worsening of the symptoms after treatment.

Studies including tests to quantify involuntary urine loss or remodelling of the pelvic structures reported improvement after laser therapy. These included the pad weight test, perineometry, Q-tip test, urodynamics and epithelial biopsies. In all studies using pad weight test there was significant decrease in mean pad weight after treatment. The Q-tip test also showed improvement in urethra angle with Valsalva manoeuvre which reveals strengthening of its supporting structures. Results concerning perineometry were not consistent. Urodynamic tests had no significant differences except in residual volume which significantly decreased. Additionally, studies comprising histopathological investigation found increased proliferative activity, remodelling of the stromal components of vaginal mucosa, higher synthesis of collagen and neoangiogenesis. ${ }^{25,26}$

The majority of the patients tolerated treatments well; few of them reported minor symptoms during laser sessions, which ranged from mild discomfort to warmth sensation. Only one patient quitted treatment due to intolerance. After sessions, few patients suffered increased vaginal discharge, spotting, vulvar oedema or burning sensation or urinary infections. Except for the last one, which eventually required antibiotics, the other symptoms were transient and self-limited. 19 patients had transient de novo UUI after laser sessions.

One of the main limitations of this review in order to take any conclusion about the efficacy and safety of laser treatments in female $\mathrm{UI}$ is the small number of studies, being the sum of 892 female patients a number with little impact to expand these results to the general population. Other limitation is that, even though the studies are prospective, there are no RCT or cost-effectiveness studies. It would be interesting to further compare laser therapy to current standard therapeutics, or to blinded control groups treated with sham. Given the importance of RCT to establish a novel treatment in clinical practice, the impact of the results of these studies and thus the conclusions of this review are limited. Other notable limitation is the lack of long-term evidence, the longest follow-up was 36 months, in only one study, ${ }^{8}$ being the remaining ones limited to a maximum of 24 months follow-up, so it is still not possible to take conclusions about potential cure, long term effectiveness, recurrence and safety. There are also no studies comparing the different number of sessions, specifically comparing the effect on patients with SUI and UUI, and patients with or without pelvic organ prolapse separately. All studies included only patients with primary UI, so there is no information about these treatments on patients previously treated with surgery and there are no studies evaluating the impact of these treatments on sex life.

About the methodologies of the studies, there is little information about who applied the questionnaires and how patients answered them, which could have implications in the results. There are also few considerations about the blinding of the subjects who performed the 
tests requiring measurements, such as the pad weight or the Q-tip, as well the histological examinations. The largest information reported is based on patient reported outcomes, which have the limitations of all subjective methods. Additionally, urodynamics (which are the current standard quantitative evaluation method of UI) were performed in only one study. It should be kept in mind that the statistical significance of an outcome does not always suggest clinical or policy relevance. Likewise, a non-significant result does not demonstrate that a treatment is ineffective, especially in studies with low power due to limited sample size.

\section{Conclusion}

Laser therapy seems to be a promising alternative approach to urinary incontinence in women. It seems effective in short term, well tolerated, with few, rare and transient side effects. However, due to the little number of studies and their low level of evidence, more research is required to further safely and effectively apply these technologies in clinical daily routine.

\section{Acknowledgments}

None.

\section{Funding information}

No funds were involved in the design and conduct of the study, collection, management, analysis and interpretation of data, preparation, review, or approval of the manuscript or in the decision to submit the manuscript for publication.

\section{Compliance with ethical standards}

\section{Conflict of interest}

The authors declare that they have no conflict of interest.

\section{Ethical approval}

This article does not contain any studies with human participants performed by any of the authors.

\section{Informed consent}

For this type of study, formal consent is not required.

\section{References}

1. Norton PBL. Urinary incontinence in women. Lancet. 2006;367(9504):5767.

2. Haylen BT, de Ridder D, Freeman RM, et al. An International Urogynecological Association (IUGA)/International Continence Society (ICS) joint report on the terminology for female pelvic floor dysfunction. Neurourol Urodyn. 2010;21(1):5-26.

3. Cervigni M, Gambacciani M. Female urinary stress incontinence Climacteric. 2015;18 Suppl 1:30-6.

4. Gaspar A, Brandi H. Non-ablative erbium YAG laser for the treatment of type III stress urinary incontinence (intrinsic sphincter deficiency). Lasers Med Sci. 2017;32(3):685-91.

5. Shamout S, Campeau L. Stress urinary incontinence in women: Current and emerging therapeutic options. Can Urol Assoc J. 2017;11 (6Supp12):S155-S158.

6. Gambacciani M, Palacios S. Laser therapy for the restoration of vaginal function. Maturitas. 2017;99:10-15.
7. Pitsouni E, Grigoriadis T, Falagas ME, et al. Laser therapy for the genitourinary syndrome of menopause. A systematic review and metaanalysis. Maturitas. 2017;103:78-88.

8. González Isaza P, Jaguszewska K, Cardona JL, et al. Long-term effect of thermoablative fractional CO2laser treatment as a novel approach to urinary incontinence management in women with genitourinary syndrome of menopause. Int Urogynecol J. 2018;29(2):211-15.

9. Tadir Y, Gaspar A, Lev-Sagie A, et al. Light and energy based therapeutics for genitourinary syndrome of menopause: Consensus and controversies. Lasers Surg Med. 2017;49(2):137-59.

10. Ogrinc UB, Senčar S, Lenasi H. Novel minimally invasive laser treatment of urinary incontinence in women. Lasers Surg Med. 2015;47(9):689-97.

11. Vizintin Z, Lukac M, Kazic M, et al. Erbium laser in gynecology. Climacteric. 2015;18 Suppl 1:4-8.

12. Pergialiotis V, Prodromidou A, Perrea DN, et al. A systematic review on vaginal laser therapy for treating stress urinary incontinence: Do we have enough evidence? Int Urogynecol J. 2017;28(10):1445-51.

13. Liberati A, Altman DG, Tetzlaff J, et al. The PRISMA statement for reporting systematic reviews and meta-analyses of studies that evaluate health care interventions: explanation and elaboration. 2009: 339:b2700.

14. Tien YW, Hsiao SM, Lee CN, et al. Effects of laser procedure for female urodynamic stress incontinence on pad weight, urodynamics, and sexual function. Int Urogynecol J. 2017;28(3):469-76.

15. Fistonić N, Fistonić I, Lukanovič A, et al. First assessment of short-term efficacy of Er:YAG laser treatment on stress urinary incontinence in women: Prospective cohort study. Climacteric. 2015;18 Suppl 1:37-42.

16. Perino A, Cucinella G, Gugliotta G, et al. Is vaginal fractional $\mathrm{CO} 2$ laser treatment effective in improving overactive bladder symptoms in postmenopausal patients? Preliminary results. Eur Rev Med Pharmacol Sci. 2016;20:2491-97.

17. Pitsouni E, Grigoriadis T, Tsiveleka A, et al. Microablative fractional CO2-laser therapy and the genitourinary syndrome of menopause: An observational study. Maturitas. 2016;94:131-36.

18. Fistonić N, Fistonić I, Guštek ŠF, et al. Minimally invasive, non-ablative Er:YAG laser treatment of stress urinary incontinence in women-a pilot study. Lasers Med Sci. 2016;31(4):635-43.

19. Pardo JI, Solà VR, Morales AA. Treatment of female stress urinary incontinence with Erbium-YAG laser in non-ablative mode. Eur J Obstet Gynecol Reprod Biol. 2016;204:1-4.

20. Gambacciani M, Levancini M, Cervigni M. Vaginal erbium laser: the second-generation thermotherapy for the genitourinary syndrome of menopause. Climacteric. 2015;18(5):757-63.

21. Lin YH, Hsieh WC, Huang L, et al. Effect of non-ablative laser treatment on overactive bladder symptoms, urinary incontinence and sexual function in women with urodynamic stress incontinence. Taiwan J Obstet Gynecol. $2017 ; 56: 815-820$

22. Fistonić I, Fistonić N. Baseline ICIQ-UI score, body mass index, age, average birth weight, and perineometry duration as promising predictors of the short-term efficacy of Er:YAG laser treatment in stress urinary incontinent women: A prospective cohort study. Lasers Surg Med. 2018.

23. Behnia-Willison F, Sarraf S, Miller J, et al. Safety and long-term efficacy of fractional CO2laser treatment in women suffering from genitourinary syndrome of menopause. Eur J Obstet Gynecol Reprod Biol. 2017;213:3944.

24. Gambacciani M, Levancini M. Short-term effect of vaginal erbium laser on the genitourinary syndrome of menopause. Minerva Ginecol. 2015;67(2):97-102. 
25. Lapii GA, Yakovleva AY, Neimark AI. Structural Reorganization of the Vaginal Mucosa in Stress Urinary Incontinence under Conditions of Er:YAG Laser Treatment. Bull Exp Biol Med. 2017;162(4):510-14.

26. Lapii GA, Yakovleva AY, Neimark AI, et al. Study of Proliferative Activity of Vaginal Epithelium in Women with Stress Urinary Incontinence Treated by Er:YAG Laser. Bull Exp Biol Med. 2017;163(2):280-83.
27. Pagano I, Gieri S, Nocera F, et al. In Oncological Patients : Preliminary Results. 2017;8(5):452-63.

28. Coyne KS, Thompson CL, Lai JS, et al. An overactive bladder symptom and health-related quality of life short-form: Validation of the OAB-q SF. Neurourol Urodyn. 2015;34(3):255-63. 